\title{
Vacunación universal contra el virus de la influenza: consenso de expertos en México
}

\author{
Guillermo M. Ruiz-Palacios y Santos ${ }^{1}$, Miguel Betancourt-Cravioto², Francisco J. Espinosa-Rosales ${ }^{3}$, \\ Rodolfo Rivas-Ruiz* ${ }^{*}$, Martha C. Guerrero-Almeida ${ }^{5}$, Ma. de Lourdes Guerrero-Almeida', \\ Marte Hernández-Porras ${ }^{6}$, Alejandro E. Macías-Hernández ${ }^{7}$, Mercedes Macías-Parra ${ }^{6}$, \\ Sarbelio Moreno-Espinosa ${ }^{8}$, Mussaret Bano-Zaidi', Daniel E. Noyola10, José Ramos-Castañeda ${ }^{11}$, \\ Norberto Reyes-Paredes ${ }^{12}$, Romeo S. Rodríguez-Suárez ${ }^{13}$, Fortino Solórzano-Santos ${ }^{14}$ y \\ Heladio G.V. Vargas-Ramírez ${ }^{15}$
}

'Departamento de Infectología, Instituto Nacional de Ciencias Médicas y Nutrición Salvador Zubirán, Ciudad de México; ${ }^{2}$ Sociedad Mexicana de Salud Pública, Cuernavaca, Morelos; ${ }^{3}$ Fundación Mexicana para Niñas y Niños con Inmunodeficiencias Primarias, AC (FUMENI), Ciudad de México; ${ }^{4}$ Centro de Adiestramiento e Investigación Clínica, División e Investigación Clínica, Instituto Mexicano del Seguro Social, Ciudad de México; ${ }^{5}$ Centro de Vacunación Prevenire, Morelia, Michoacán; ${ }^{6}$ Departamento de Infectología, Instituto Nacional de Pediatría, Ciudad de México; ${ }^{7}$ Departamento de Infectología, Universidad de Guanajuato, Guanajuato; ${ }^{8}$ Departamento de Infectología, Hospital Infantil de México Federico Gómez, Ciudad de México; 'Unidad de Investigación de Enfermedades Infecciosas, Hospital General Dr. Agustín O'Horán, Mérida, Yucatán; ${ }^{10}$ Departamento de Microbiología, Facultad de Medicina, Universidad Autónoma de San Luis Potosí, San Luis Potosí; ${ }^{11}$ Centro de Investigaciones sobre Enfermedades Infecciosas, Instituto Nacional de Salud Pública, Cuernavaca, Morelos; ${ }^{12}$ Servicio de Ginecología y Obstetricia, Instituto Nacional de Perinatología, Ciudad de México; ${ }^{13}$ Fundación IMSS AC, Ciudad de México; ${ }^{14}$ Unidad de Investigación en Medicina basada en Evidencias, Hospital Infantil de México Federico Gómez, Ciudad de México; ${ }^{15}$ Departamento de Pediatría, Hospital General de Zacatecas, Zacatecas. México

\section{Resumen}

La influenza es una enfermedad costosa para la población. Es causa de morbimortalidad estacional, epidemias y pandemias o sindemias. Debido a la variabilidad del virus, se implementan sistemas de vigilancia para actualizar las cepas e incluirlas en la vacuna antiinfluenza anual. Actualmente se recomienda esta vacuna en algunos grupos de alto riesgo. Sin embargo, la vacunación universal es aún controvertida. Objetivo: Evaluar la evidencia y describir la posición de un panel de expertos sobre la pertinencia de la vacunación universal contra el virus de influenza. Material y métodos: Se realizaron cinco preguntas clínicas, con las que se realizó una búsqueda sistemática de la literatura en fuentes electrónicas y un panel Delphi. Se analizó la evidencia y se emitieron recomendaciones por los expertos. Resultados: El grupo de expertos recomienda vacunar a la población desde los seis meses de edad e incluir a personas que viven con alergia a la proteína del huevo, con comorbilidades (diabetes, obesidad, cáncer), trabajadores de la salud y embarazadas. Conclusiones: La vacunación, iniciando con los grupos vulnerables, es una estrategia necesaria, ética y costo-efectiva. Sin embargo, extender la cobertura para lograr la vacunación universal podría disminuir la transmisión de la enfermedad y sus consecuencias en la población.

PALABRAS CLAVE: Influenza. Vacunación. Grupos de riesgo. Embarazo. Profesionales de la salud.

\section{Universal influenza vaccination: a Mexican Expert Position Paper}

\section{Abstract}

Influenza is a costly disease for the population. It is a cause of seasonal morbidity and mortality, epidemics and pandemics or syndemics. Given the variability of the virus, surveillance systems are implemented in order to update the strains and include them in the annual influenza vaccine. This vaccine is currently recommended in some high-risk groups. However, universal

Correspondencia:

${ }^{*}$ Rodolfo Rivas-Ruiz

E-mail: rivasrodolfo@gmail.com
Gac Med Mex. 2021;157:651-656

Disponible en PubMed

www.gacetamedicademexico.com

0016-3813/@ 2021 Academia Nacional de Medicina de México, A.C. Publicado por Permanyer. Este es un artículo open access bajo la licencia CC BY-NC-ND (http://creativecommons.org/licenses/by-nc-nd/4.0/). 
vaccination remains controversial. Objective: To evaluate the evidence and describe the position of a panel of experts on the relevance of universal vaccination against influenza virus. Material and methods: Five clinical questions were asked, whereby a systematic search of the literature in electronic sources and a Delphi panel was carried out. The evidence was analyzed and recommendations were issued by the experts. Results: The group of experts recommends vaccinating the population starting at six months of age include people who live with egg protein allergy; with comorbidities (diabetes, obesity, cancer) health workers and pregnant women. Conclusions: Vaccination, starting with vulnerable groups, is a necessary, ethical and cost-effective strategy. However, extending coverage to achieve universal vaccination could reduce the transmission of the disease and its consequences in the population.

KEY WORDS: Influenza. Vaccination. Risk groups. Pregnancy. Health professionals.

\section{Introducción}

Las infecciones causadas por el virus de la influenza afectan al $5-30 \%$ de la población, lo que supone de 3 a 5 millones de casos de enfermedad grave y 290,000 a 650,000 muertes en el mundo'. La vacunación es la forma más efectiva para prevenir la enfermedad y sus consecuencias ${ }^{1,2}$.

La vacuna ha demostrado la disminución de la enfermedad moderada a grave, las hospitalizaciones y su transmisión. Se ha determinado que la eficacia de la vacuna es del $59 \%$ (intervalo de confianza del $95 \%$ [IC 95\%]: $52-72 \%$ ) en los niños y del $65 \%$ (IC 95\%: 54-73) en los adultos > 65 años de edad ${ }^{3,4}$.

Con la llegada de la pandemia producida por el coronavirus 2 del síndrome respiratorio agudo grave (SARSCoV-2) se hizo patente la sindemia que corresponde a la interacción entre dos o más epidemias/pandemias presentes al mismo tiempo en una población $n^{5}$. En nuestro país esta sindemia corresponde a la interacción provocada por la pandemia por el SARS-CoV-2, la epidemia de obesidad, diabetes y otras comorbilidades, así como la constante presencia de la influenza. El combate de esta sindemia requiere de la mayor cantidad de acciones simultáneas, entre las que se encuentra la vacunación universal contra la influenza.

En México, la estrategia de vacunación contra influenza está centrada en grupos considerados de alto riesgo para el desarrollo de complicaciones. Sin embargo, aún es controvertido el ampliar la cobertura a otros grupos poblacionales, por lo que se convocó a expertos en el tema para evaluar la evidencia y emitir un consenso sobre la pertinencia de la vacunación universal.

\section{Metodología}

Se convocó a un grupo multidisciplinario de expertos en vacunación de influenza en México. Utilizando la metodología Delphi, se diseñó un cuestionario que fue enviado al grupo de expertos para obtener un preconsenso acerca de los aspectos que discutir sobre la vacunación universal.

Durante la reunión presencial se mostró al grupo el resultado del cuestionario Delphi. Se realizó una búsqueda sistemática de la literatura en bases de datos electrónicas (PubMed, Bireme y Google Scholar) por cada uno de los temas usando el acrónimo PICO (pacientes, intervención/comparación y resultados [outcomes]). Cada equipo revisó todos los artículos obtenidos. Los equipos presentaron al pleno el resultado de su trabajo, en el cual se tomó en cuenta la metodología y las medidas de relevancia clínica. En 15 de 27 temas (56\%) se alcanzó consenso, por lo que en la discusión se enfatizaron los temas en los que no se logró llegar a un acuerdo. En esos casos, los temas fueron revisadas hasta llegar a un consenso. Después de la reunión, el editor encargado reunió los textos de cada equipo para conformar una primera versión del documento, mismo que fue discutido en pleno en una segunda reunión para obtener la versión final.

\section{Resultados}

\section{Ampliar del esquema de vacunación a los niños entre 5 y 12 años}

\section{RECOMENDACIÓN}

La vacuna contra la influenza debe abarcar el grupo de edad de los 6 meses hasta los 12 años, con o sin factores de riesgo.

\section{Fundamento}

La recomendación de ampliar los grupos de edad para incluir al grupo de niños entre 5 y 12 años tiene 
como base que entre ellos se generan los niveles más altos de transmisión de la influenza ${ }^{6}$. Cuando se logra reducir la tasa de infección en los niños de edad escolar, mediante un incremento de la cobertura, no solo disminuye el riesgo de contagios (razón de momios [RM]: 0.7; IC 95\%: 0.62-0.89), sino que también baja la carga de enfermedad en otras poblaciones no contempladas en el esquema de vacunación ${ }^{3}$. La inmunización de los escolares podría reducir la frecuencia de casos de influenza entre los contactos intradomiciliarios.

\section{Vacunación en población con antecedente de alergia a la proteína del huevo}

\section{RECOMENDACIÓN}

Los niños con alergia a la proteína del huevo (APH) deben ser vacunados en una unidad de salud que cuente con capacidad de respuesta para el manejo de una posible reacción anafiláctica y que siga las guías internacionales de vacunación. La vacuna solo está contraindicada en sujetos con antecedente de reacción anafiláctica previa.

\section{FUNDAMENTO}

La prevalencia actual de la APH en México $(0.4 \%$ de la población) es baja ${ }^{7}$. La frecuencia reportada de anafilaxia es de 1.3 casos $/ 1,000,000$. La mayoría de estos eventos ocurrió en pacientes con historia documentada de reacciones alérgicas graves al huevo ${ }^{8}$. Las reacciones anafilácticas relacionadas con las vacunas son raras debido al bajo contenido de ovoalbúmina $(<1 \mathrm{mg} / \text { dosis })^{9}$. Sin embargo, se recomienda que se administre la vacuna en hospitales ${ }^{10}$.

\section{Vacunación contra influenza durante el embarazo}

\section{RECOMENDACIÓN}

- La vacuna inactivada debe aplicarse en todas las mujeres embarazadas, independientemente del trimestre de gestación.

- Las mujeres que no se vacunaron durante el embarazo deben recibir una dosis en el periodo posparto o durante la lactancia.

\section{Fundamentos}

La vacuna inactivada contra la influenza ha mostrado ser segura para la madre y el feto. La vacuna contra la influenza no incrementa la muerte fetal, malformaciones congénitas 0 abortos espontáneos ${ }^{11}$. Es importante hacer notar que la administración de vacunas de virus vivos está contraindicada durante el embarazo ${ }^{12}$.

Debido a que la vacuna contra influenza no se recomienda para $<6$ meses de edad, la vacuna debe aplicarse al menos cuatro semanas antes del nacimiento ${ }^{12}$. Ensayos clínicos controlados han ratificado la eficacia de la vacunación en embarazadas del 27 al $63 \%$ para prevenir la influenza confirmada por laboratorio y del $29 \%$ para enfermedad respiratoria aguda en el recién nacido y lactantes, como previamente lo sugerían estudios no aleatorizados ${ }^{13,14}$. De acuerdo con modelos predictivos de nuestro país, para disminuir la mortalidad materno-fetal se sugiere una cobertura con vacuna de influenza mínima del $80 \%$ de las embarazadas y vacunación individual de mujeres en edad fértil ${ }^{12}$.

\section{Vacunación del personal de salud en unidades de atención médica}

\section{RECOMENDACIÓN}

Todos los trabajadores de la salud deben recibir la vacuna, de forma universal y anualmente.

\section{FundAMENTO}

Los brotes de influenza nosocomial son frecuentes y alcanzan una tasa de ataque que oscila entre el 12 y $60 \%{ }^{15}$.

Entre los factores más importantes que contribuyen a los brotes nosocomiales de influenza destacan una baja tasa de vacunación y poco apego entre los trabajadores de la salud a las medidas de higiene ${ }^{16}$. Aunado al efecto de la pandemia que provocó una disminución del $4.2 \%$ en la vacunación del personal de salud ${ }^{17}$. En México la adherencia a la vacunación contra la influenza es del $63.5 \%$ en los trabajadores sanitarios, la cual es considerada subóptima ${ }^{18}$. Lo anterior debido a que se ha demostrado la eficacia de la vacuna (70 al $90.4 \%$ ) para reducir el número de infecciones por influenza en trabajadores de salud vacunados contra la influenza $A(\mathrm{H} 1 \mathrm{~N} 1)^{19,20}$. 
Aún se debate acerca de las políticas obligatorias de vacunación contra la influenza para aumentar las tasas de vacunación entre el personal de salud y se hacen esfuerzos para poder implementarlas en los hospitales ${ }^{21}$. El beneficio de la vacunación en el personal de salud se extiende a una disminución en las tasas de infección, en los días de ausentismo y en los costos de atención ${ }^{22}$.

\section{Influenza y comorbilidades}

\section{RECOMENDACIÓN}

Todos los pacientes con comorbilidades, en especial diabetes mellitus (DM), asma, enfermedad pulmonar obstructiva crónica (EPOC), enfermedad renal, sobrepeso y obesidad, enfermedades cardiovasculares e inmunocomprometidos deben recibir la vacuna contra influenza anualmente.

\section{FundAMENTOS}

Al ser una enfermedad inflamatoria, la infección por influenza en pacientes con comorbilidades incrementa el riesgo de hospitalización y muerte ${ }^{23}$. Las personas que viven con diabetes se benefician de la vacunación para prevenir la hospitalización y la neumonía causadas por el virus de la influenza y esta enfermedad, independientemente de la edad y del estado de control metabólico que tengan ${ }^{24}$.

Así mismo, el riesgo de hospitalización por influenza es mayor en los pacientes con enfermedad cardiovascular (RM: 1.63; IC 95\%: 1.33-2.02) ${ }^{25}$, asma (RM: 2.25; IC 95\%: 1.67-3.03) ${ }^{26}$, cáncer (RM: 2.25; IC 95\%: 1.234.11) ${ }^{27}$, enfermedad renal (RM: 2.11; IC 95\%: 1.48$3.01)^{28}$, obesidad (IMC > 30; RM: 3.28; IC 95\%: 1.73-5.91), como en las personas que padecen obesidad mórbida (RM: 18.4; IC 95\%: 7.83-47.4) ${ }^{29}$. Los pacientes obesos tienen mayor susceptibilidad y peor pronóstico tanto en la infección por influenza como por SARS-CoV-2 $2^{30,31}$

Además de prevenir la muerte, la vacuna previene el riesgo de infarto de miocardio agudo entre un 15 y $45 \%$ en población general ${ }^{32}$. En pacientes con enfermedad coronaria, la vacuna redujo la mortalidad cardiovascular (riesgo relativo [RR]: 0.45; IC 95\%: 0.26-0.76) ${ }^{33,34}$.

No hay información contundente sobre el beneficio de la vacuna de influenza en pacientes con EPOC y asma. Sin embargo, su uso se ha asociado a una disminución de exacerbaciones y hospitalizaciones ${ }^{35}$.
Tabla 1. Población con comorbilidades que requiere ser vacunada contra influenza

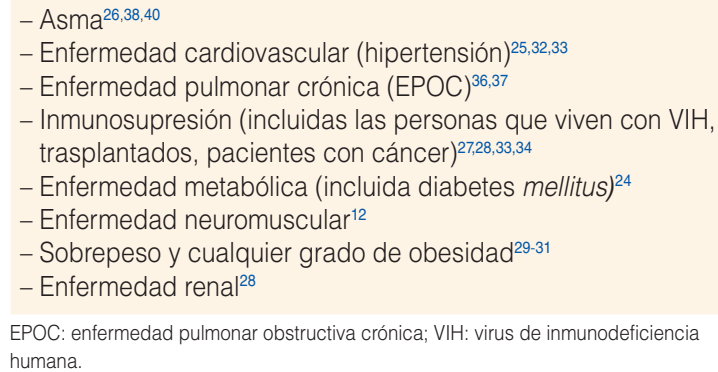

Si bien la información no es suficiente, los pacientes con EPOC vacunados contra influenza tienen un $37 \%$ menos exacerbaciones que los no vacunados. El riesgo estimado es de 0.82 (IC 95\%: 0.79-0.96) para evitar la insuficiencia respiratoria en pacientes con EPOC vacunados contra la influenza ${ }^{36}$, esto resulta en una disminución del $90 \%$ del costo de la atención de las exacerbaciones de EPOC asociadas a hospitalización, por lo que la vacuna es costo-benéfica ${ }^{37}$.

En los pacientes con asma se ha observado que las hospitalizaciones por exacerbaciones de asma aumentan durante la estación de influenza ${ }^{38}$. Se ha considerado que la vacunación para prevenir la influenza puede ser un factor protector en asmáticos, aunque los resultados no son concluyentes. En la práctica, la mejor evolución de los asmáticos vacunados que fueron hospitalizados por exacerbación sugiere que la vacunación brinda cierta protección (Tabla 1) $)^{39,40}$.

\section{Vacunación en pacientes que viven con cáncer}

\section{RECOMENDACIÓN}

Todos los pacientes con cáncer, independientemente de la etapa de tratamiento en que se encuentren, deben recibir la vacuna contra influenza.

\section{FUNDAMENTOS}

Se ha demostrado beneficio de la vacunación contra influenza en pacientes con neoplasias independientemente del tipo de cáncer y tratamiento.

Se ha demostrado su beneficio en pacientes con cáncer de colon y recto (RR: $0.5 ; p<0.001$ ) y en pulmón (RR: 0.428; $p=0.002)^{41}$. En pacientes con 
tumores sólidos que reciben quimioterapia se ha demostrado disminución de la mortalidad (RM: 0.88; IC 95\%: 0.77-0.99 ${ }^{42}$. Además, reduce el número de interrupciones del tratamiento oncológico, independientemente del momento del ciclo de quimioterapia en el que se aplicó la vacuna ${ }^{43}$.

\section{Discusión}

Hemos discutido la evidencia actual y consideramos que las recomendaciones arriba realizadas son sólidas y podría traer beneficios en general a la población mexicana.

Además, la vacuna de influenza ha demostrado ser costo-efectiva al disminuir la mortalidad, los ingresos a hospitalización y la calidad de vida.

Se estimó que, en 2018, los costos médicos directos se estimaron en \$ 3.2 mil millones (\$1.5- \$ 11.7 mil millones) y los costos indirectos en $\$ 8.0$ mil millones (\$ 4.8- \$13.6 mil millones) ${ }^{44}$.

En nuestro país un estudio transversal de costo-efectividad mostró que lograr la cobertura de vacunación universal podría prevenir 154,143 ingresos a las salas de emergencias y 97,637 hospitalizaciones, con un ahorro anual de 3,90 a 111,99 millones de $\mathrm{USD}^{45}$.

El consenso de los expertos determinó que es recomendable la ampliación de la vacunación en los niños escolares y adolescentes. Esto es similar a lo que se ha recomendado en otros países como Japón ${ }^{6}$. En México se demostró que la ampliación de la vacunación a este grupo de niños es una acción costo-efectiva que podría ahorrar hasta 111.9 millones de dólares ${ }^{46}$.

Es importante reconocer que por cuestiones económicas se deben considerar factores específicos para priorizar la inclusión paulatina de toda la población. El grupo de expertos se adhiere a la recomendación universal propuesta por las agencias de salud internacionales y en el caso del Sector Público en México, solicita que en la medida de lo posible se cuente con un presupuesto que permita cuando menos cumplir el objetivo de ampliar la vacunación contra influenza de los 6 meses a los 12 años de vida y a los grupos con comorbilidades en mayor riesgo.

Finalmente, este grupo de expertos acentúa la necesidad de la vacunación contra influenza previa a la próxima estación 2022-2023, ya que durante la pandemia producida por el SARS-CoV-2, la prevalencia de la mayoría de los virus respiratorios que comúnmente circulan y causan enfermedad respiratoria
Tabla 2. Grupos recomendados que deben recibir la vacuna contra influenza

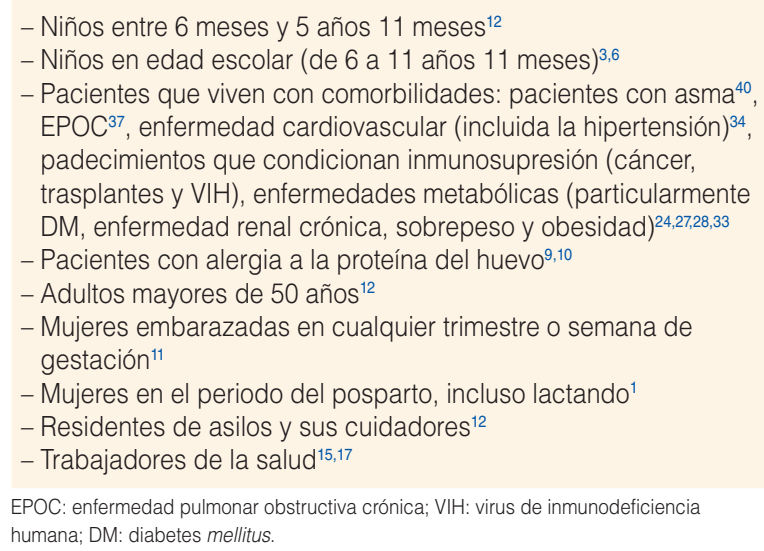

aguda ha sido mínima o nula y no se sabe cuál será el curso de la reemergencia de influenza en un futuro cercano y sus consecuencias en una población cuyas defensas contra la influenza muy probablemente han disminuido.

Con base en las evidencias científicas actuales, los grupos recomendados como prioritarios que deberían recibir la vacuna contra influenza se describen en la tabla 2.

\section{Agradecimientos}

Los autores agradecen a los participantes en este panel, que ayudaron en cada una de las etapas del Panel Delphi y a los bibliotecarios que ayudaron con la bibliografía.

\section{Financiamiento}

Este trabajo fue financiado por Sanofi Pasteur, México. El patrocinador no tuvo ningún papel en el diseño del manuscrito, ni en la recopilación, el análisis y la interpretación de los datos, ni en la redacción del manuscrito.

\section{Conflicto de intereses}

Los autores declaran no tener conflicto de intereses.

\section{Responsabilidades éticas}

Protección de personas y animales. Los autores declaran que para esta investigación no se han 
realizado experimentos en seres humanos ni en animales.

Confidencialidad de los datos. Los autores declaran que han seguido los protocolos de su centro de trabajo sobre la publicación de datos de pacientes.

Derecho a la privacidad y consentimiento infor-

mado. Los autores declaran que en este artículo no aparecen datos de pacientes.

\section{Bibliografía}

1. Qualls N, Levitt A, Kanade N, Wright-Jegede N, Dopson S, Biggerstaff M et al. Community Mitigation Guidelines to Prevent Pandemic Influenza - United States, 2017. MMWR Recomm Reports. 2017;66(1):1-34.

2. Grohskopf LA, Sokolow L, Broder K, Walter EB, Fry AM, Jernigan DB Prevention and control of seasonal influenza with vaccines: Recommendations of the Advisory Committee on Immunization Practices - United States, 2018-19 Influenza Season. MMWR Recomm Reports. 2018;67(03):1-20.

3. Sánchez-Ramos EL, Monárrez-Espino J, Noyola DE. Impact of vaccination on influenza mortality in children. Vaccine. 2017;35(9):1287-92.

4. Tricco AC, Chit A, Soobiah C, Hallett D, Meier G, Chen MH, et al. Comparing influenza vaccine efficacy against mismatched and matched strains: a systematic review and meta-analysis. BMC Med. 2013;11:153.

5. Antonio-Arques V, Franch-Nadal J, Caylà JA. Diabetes and tuberculosis: a syndemic complicated by COVID-19. Med Clin (Barc). 2021:S00257753(21)00208-6.

6. Reichert TA, Sugaya N, Fedson DS, Glezen WP, Simonsen L, Tashiro M The Japanese Experience with vaccinating schoolchildren against Influenza. N Engl J Med. 2001;344(12):889-96.

7. Góngora-Meléndez MA, Magaña-Cobos A, Montiel-Herrera JM, Pantoja-Minguela CL, Pineda-Maldonado ML, Piñeyro-Beltrán EE. Alergia a las proteínas del huevo en edad pediátrica. Rev Alerg Mex. 2015;62(3):234-50

8. Moro PL, Woo EJ, Marquez P, Cano M. Monitoring the safety of high-dose, trivalent inactivated influenza vaccine in the vaccine adverse event reporting system (VAERS), 2011 - 2019. Vaccine. 2020:38(37):5923-6.

9. Des Roches A, Paradis L, Gagnon R, Lemire C, Bégin P, et al.; PCIRN (Public Health Agency of Canada/Canadian Institutes of Health Research Influenza Research Network). Egg-allergic patients can be safely vaccinated against influenza. J Allergy Clin Immunol. 2012;130(5):1213-6.

10. Greenhawt M, Turner PJ, Kelso JM. Administration of influenza vaccines to egg allergic recipients: A practice parameter update 2017. Ann Allergy Asthma Immunol. 2018;120(1):49-52.

11. Macias AE, Precioso AR, Falsey AR, Global Influenza Initiative. The Global Influenza Initiative recommendations for the vaccination of pregnant women against seasonal influenza. Influenza Other Respi Viruses. 2015;9(Suppl 1):31-7

12. Secretaría de Salud. Conoce los grupos prioritarios para vacunarse contra la influenza [Internet]. Gobierno de México, Secretaría de Salud [citado: enero 2021]. Disponible en: https://www.gob.mx/salud/articulos/ conoce-los-grupos-prioritarios-para-vacunarse-contra-la-influenza.

13. Tapia MD, Sow SO, Tamboura B, Tégueté I, Pasetti MF, Kodio M, et al. Maternal immunisation with trivalent inactivated influenza vaccine for prevention of influenza in infants in Mali: a prospective, active-controlled observer-blind, randomised phase 4 trial. Lancet Infect Dis. 2016:16(9):1026-35

14. Steinhoff MC, Katz J, Englund JA, Khatry SK, Shrestha L, Kuypers J, et al. Year-round influenza immunisation during pregnancy in Nepal: a phase 4 randomised, placebo-controlled trial. Lancet Infect Dis. 2017;17(9):981-9.

15. Javaid W, Ehni J, Gonzalez-Reiche AS, Carreño JM, Hirsch E, Tan J, et al. Real-time investigation of a large nosocomial influenza A outbreak informed by genomic epidemiology. Clin Infect Dis. 2020;30:ciaa1781.

16. Vanhems $P$, Bénet $T$, Munier-Marion E. Nosocomial influenza: encouraging insights and future challenges. Curr Opin Infect Dis. 2016;29(4):366-72.

17. Kang M, Clark S, Mendoza S, Arocha D, Cutrell JB, Perl TM, et al. Influenza vaccination among healthcare personnel during the coronavirus disease 2019 (COVID-19) pandemic. Infect Control Hosp Epidemiol. $2021 ; 9: 1-2$

18. Ochoa-Hein E, Gutiérrez-López EN, Torres-Erazo DS, Núñez-Caamal NJ, Martínez-Longoria CA, García-Bonilla LA, et al. Factors associated with influenza vaccination acceptance in Mexican healthcare workers: A multicenter cross-sectional study. Prev Med. 2021:148:106560.

19. Dini G, Toletone A, Sticchi L, Orsi A, Bragazzi NL, Durando P. Influenza vaccination in healthcare workers: A comprehensive critical appraisal of the literature. Hum Vaccin Immunother. 2018;14(3):772-89.
20. Maltezou HC, Theodoridou K, Ledda C, Rapisarda V, Theodoridou M. Vaccination of healthcare workers: is mandatory vaccination needed? Expert Rev Vaccines. 2019;18(1):5-13.

21. Stead M, Critchlow N, Eadie D, Sullivan F, Gravenhorst K, Dobbie F. Mandatory policies for influenza vaccination: Views of managers and healthcare workers in England. Vaccine. 2019;37(1):69-75

22. Laris González A, Villa Guillén M, López Martínez B, Gamiño Arroyo AE, Moreno Espinosa S, Jiménez Juárez RN, et al. Influenza-like illness in healthcare personnel at a paediatric referral hospital: Clinical picture and impact of the disease. Influenza Other Respi Viruses 2018;12(4):475-81.

23. Lansbury LE, Brown CS, Nguyen-Van-Tam JS. Influenza in long-term care facilities. Influenza Other Respi Viruses. 2017:11(5):356-66.

24. Remschmidt C, Wichmann O, Harder T. Vaccines for the prevention of seasonal influenza in patients with diabetes: systematic review and meta-analysis. BMC Med. 2015;13:53.

25. Clar C, Oseni Z, Flowers N, Keshtkar-Jahromi M, Rees K. Influenza vaccines for preventing cardiovascular disease. Cochrane Database Syst Rev. 2018;(5):CD005050.

26. Cates CJ, Rowe BH. Vaccines for preventing influenza in people with asthma. Cochrane Database Syst Rev. 2013;(2):CD000364

27. Eliakim-Raz N, Vinograd I, Zalmanovici Trestioreanu A, Leibovici L, Paul M. Influenza vaccines in immunosuppressed adults with cancer. Cochrane Database Syst Rev. 2013;10:CD008983.

28. Ma BM, Yap DYH, Yip TPS, Hung IFN, Tang SCW, Chan TM. Vaccination in patients with chronic kidney disease-Review of current recommendations and recent advances. Nephrology (Carlton). 2021;26(1):5-11.

29. Moser JS, Galindo-Fraga A, Ortiz-Hernández AA, Gu W, Hunsberger S, Galán-Herrera JF, et al.; La Red ILI 002 Study Group. Underweight, overweight, and obesity as independent risk factors for hospitalization in adults and children from influenza and other respiratory viruses. Influenza Other Respir Viruses. 2019;13(1):3-9.

30. Murphy R, Fragaszy EB, Hayward AC, Warren-Gash C. Investigating obesity as a risk factor for influenza-like illness during the $2009 \mathrm{H} 1 \mathrm{~N} 1$ influenza pandemic using the Health Survey for England. Influenza Other Respi Viruses. 2017;11(1):66-73

31. Guglielmi V, Colangeli L, D'Adamo M, Sbraccia P. Susceptibility and severity of viral infections in obesity: Lessons from influenza to COVID-19. Does leptin play a role? Int J Mol Sci. 2021;22(6):3183.

32. Maclntyre CR, Mahimbo A, Moa AM, Barnes M. Influenza vaccine as a coronary intervention for prevention of myocardial infarction. Heart. 2016;102(24):1953-6.

33. Clar C, Oseni Z, Flowers N, Keshtkar-Jahromi M, Rees K. Influenza vaccines for preventing cardiovascular disease. Cochrane Database Syst Rev. 2018;5:CD005050.

34. Raffoul M, Lin KW. Influenza vaccination for the prevention of cardiovascular disease. Am Fam Physician. 2016:93(5):357-8.

35. Bekkat-Berkani R, Wilkinson T, Buchy P, Dos Santos G, Stefanidis D, Devaster J-M, et al. Seasonal influenza vaccination in patients with COPD: a systematic literature review. BMC Pulm Med. 2017:17(1):79.

36. Kopsaftis Z, Wood-Baker R, Poole P. Influenza vaccine for chronic obstructive pulmonary disease (COPD). Cochrane Database Syst Rev. 2018;6:CD002733.

37. Huang $\mathrm{HH}$, Chen SJ, Chao TF, Liu CJ, Chen TJ, Chou P, et al. Influenza vaccination and risk of respiratory failure in patients with chronic obstructive pulmonary disease: A nationwide population-based case-cohort study. J Microbiol Immunol Infect. 2019:52(1):22-9.

38. Gerke AK, Yang M, Tang F, Foster ED, Cavanaugh JE, Polgreen PM Association of hospitalizations for asthma with seasonal and pandemic influenza. Respirology. 2014;19(1):116-21.

39. Suárez-Varela MM, Llopis A, Fernandez-Fabrellas E, Sanz F, Perez-Lozano MJ, Martin V, et al. Asthma and influenza vaccination in elderly hospitalized patients: Matched case-control study in Spain. J Asthma. 2018;55(4):391-401.

40. Vasileiou E, Sheikh A, Butler C, El Ferkh K, von Wissmann B, McMenamin J, et al. Effectiveness of influenza vaccines in asthma: A systematic review and meta-analysis. Clin Infect Dis. 2018;65(8):1388-95.

41. Bitterman R, Eliakim-Raz N, Vinograd I, Zalmanovici Trestioreanu A Leibovici L, Paul M. Influenza vaccines in immunosuppressed adults with cancer. Cochrane Database Syst Rev 2018;2(10):CD008983

42. Vollaard A, Schreuder I, Slok-Raijmakers L, Opstelten W, Rimmelzwaan G, Gelderblom H. Influenza vaccination in adult patients with solid tumours treated with chemotherapy. Eur J Cancer. 2017;76:134-43.

43. Lehrer S, Rosenzweig KE. Influenza vaccination in patients with cancer. Cancer. 2017:123(7):1272-3.

44. Putri WCWS, Muscatello DJ, Stockwell MS, Newall AT. Economic burden of seasonal influenza in the United States. Vaccine. 2018;36(27):3960-6.

45. Tapia-Conyer R, Betancourt-Cravioto M, Montoya A, Falcón-Lezama JA, Alfaro-Cortes MM, Saucedo-Martínez R. A call for a reform of the influenza immunization program in Mexico: Epidemiologic and economic evidence for decision making. Vaccines (Basel). 2021;9(3):286.

46. Falcón-Lezama JA, Saucedo-Martínez R, Betancourt-Cravioto M, Alfaro-Cortes MM, Bahena-González RI, Tapia-Conyer R. Influenza in the school-aged population in Mexico: burden of disease and cost-effectiveness of vaccination in children. BMC Infect Dis. 2020;20(1):240. 